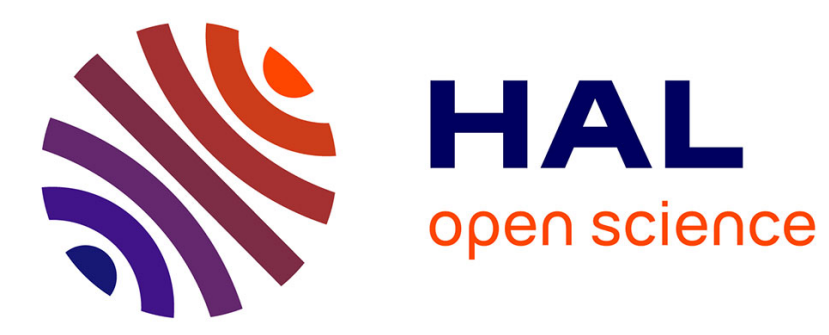

\title{
Chronique des élections de 2010 à Zanzibar
}

Marie-Aude Fouéré

\section{To cite this version:}

Marie-Aude Fouéré. Chronique des élections de 2010 à Zanzibar. Politique africaine, 2011, 121 (1), 10.3917/polaf.121.0127 . halshs-01493054

\section{HAL Id: halshs-01493054 https://shs.hal.science/halshs-01493054}

Submitted on 13 Apr 2017

HAL is a multi-disciplinary open access archive for the deposit and dissemination of scientific research documents, whether they are published or not. The documents may come from teaching and research institutions in France or abroad, or from public or private research centers.
L'archive ouverte pluridisciplinaire HAL, est destinée au dépôt et à la diffusion de documents scientifiques de niveau recherche, publiés ou non, émanant des établissements d'enseignement et de recherche français ou étrangers, des laboratoires publics ou privés. 


\title{
Chronique des élections de 2010 à Zanzibar
}

\begin{abstract}
À Zanzibar, l'année électorale 2010 semble ouvrir une nouvelle ère politique et sociale. Grâce à la formation d'un gouvernement d'unité nationale, les îles ont réussi à contenir les tensions politiques et à mettre $\mathrm{n}$ aux violences électorales observées depuis la réintroduction du multipartisme en 1992. Ce gouvernement entérine le principe du partage du pouvoir entre les deux principaux partis politiques, l'ancien parti unique du CCM, toujours au pouvoir, et le parti d'opposition du CUF. Les élections générales d'octobre se sont déroulées dans le calme et la bonne humeur malgré les soupçons de manipulations des listes électorales au moment des inscriptions et de fraudes durant le scrutin. Pourtant, les scores serrés des deux principaux candidats à la présidence témoignent de la reconduction de divisions politiques anciennes, qui superposent aux af liations partisanes des référents identitaires différents et des conceptions alternatives de l'appartenance à la nation. Le rôle de la République unie de Tanzanie dans la vie politique des îles reste aussi un élément clé pour comprendre la reconduction du CCM au pouvoir. Si le principe du partage du pouvoir est le gage de démocratisation attendu par la communauté internationale pour renouveler son aide au développement, il a ceci de stratégique qu'il permet au CCM de se maintenir au pouvoir et d'affaiblir la charge critique de l'opposition.
\end{abstract}

\begin{abstract}
Amani na utulivu. Paix et tranquillité. En République unie de Tanzanie, cette rengaine électorale a scandé l'année 2010, marquée par la tenue des quatrièmes élections générales multipartites au mois d'octobre ${ }^{1}$. Sur les affiches placardées en ville, dans les spots publicitaires à la radio et à la télévision, dans les émissions politiques retransmises aux heures de grande audience, dans les reportages télévisés du journal de 20 heures, ou encore dans les discours publics des candidats de tout bord, ces termes sont revenus sans cesse pour appeler à la tenue d'élections dans le calme et le respect des principes démocratiques. À Zanzibar ${ }^{2}$ plus que dans la partie continentale de la Tanzanie, ces termes résonnaient avec un timbre tout particulier. Depuis la réintroduction du multipartisme en $1992^{3}$, les îles de Zanzibar, qui possèdent depuis 1964 leur propre gouvernement, le Gouvernement révolutionnaire de Zanzibar, et une Chambre des représentants, sont divisées entre deux principauxpartispolitiques:l'ancienpartiuniquequisemaintientaupouvoir jusqu'à aujourd'hui, le CCM (Chama Cha Mapinduzi, « Parti de la Révolution »), et le parti d'opposition CUF (Civic United Front, « Front civique uni »), aussi appelé Chama Cha Wananchi («Parti des citoyens»). Les élections de 1995, 2000 et 2005 se sont déroulées dans un climat de tensions et de violences accrues: les fraudes électorales ont culminé dans des violences policières et militaires qui, en 2001, ont fait entre 30 et 60 morts et plus de 300 blessés, chassant vers le Kenya environ 2 300 insulaires ${ }^{4}$ et, en 2005, plus de 30 morts et des centaines de blessés ${ }^{5}$. Quant au CUF, il conteste à chaque élection la victoire du CCM et refuse de reconnaître la
\end{abstract}


légitimité du gouvernement des îles et de son président ${ }^{6}$.

Mais l'année 2010 marque une rupture avec les trois élections précédentes. Au mois de novembre 2009, un rapprochement inattendu s'est opéré entre le secrétaire général du CUF et candidat présidentiel depuis 1995, Seif Sharif Hamad, et le président en fonction de Zanzibar, Amani Abeid Karume. Les promesses de changement à l'échelle des îles faites par ces deux grandes figures

1. Cette recherche a bénéficié d'une bourse de recherche de l'Institut français de recherche en Afrique de Nairobi, dans le cadre d'un projet collectif sur les élections de 2010 en Tanzanie. L'auteure était présente à Zanzibar en décembre 2009, puis en juillet, août, octobre et novembre 2010. Aidée par un assistant de recherche pendant la phase d'inscription sur les listes électorales, elle a mené ses propres observations de terrain lors des meetings politiques publics, dans les bureaux de vote, à l'annonce des résultats, au moment des célébrations officielles postélectorales, et lors des rassemblements populaires. Elle a conduit des entretiens avec des hommes politiques, des militants, des journalistes tanzaniens et des observateurs électoraux. Assimilée à une journaliste, parlant couramment swahili, l'auteure a bénéficié d'un accès aisé au terrain, facilité par la volonté de transparence du processus électoral affichée par les autorités publiques.

2. Zanzibar est un archipel de l'océan Indien au large de la côte de la Tanzanie, composé de deux îles principales, Unguja et Pemba. Zanzibar jouit d' un statut de semi-autonomie au sein de la République unie de Tanzanie, formée en avril 1964 de l'union des deux gouvernements alors indépendants de Zanzibar et du Tanganyika.

3. Un système multipartite était en place entre 1957 et 1964 . Le système de parti unique a été instauré en janvier 1964, quelques jours après la Révolution qui a renversé le premier gouvernement indé- pendant. Mené par l'Afro-Shirazi Party, devenu Chama Cha Mapinduzi (CCM) en 1977, il a perduré jusqu'en 1992.

4. Human Right Watch, «The Bullets were Raining. The January 2001 Attack on Peaceful Demonstrators in Zanzibar », Human Right Watch Report, vol. 14, $\mathrm{n}^{\circ}$ 3(A), 2002. Les chiffres varient selon les sources. 5. Voir B. Rawlence, «Briefing: The Zanzibar Election», African Affairs, vol. 104, n 416, 2005, p. 515-523; National Democratic Institute, International Observation Mission: 2005 Zanzibar Elections. October, 30 2005. Final Report, Dar es Salaam, NDI, 2005.

6. En 1995, le décompte des voix fait planer le doute sur le respect des règles électorales : Seif Sharif Hamad (du CUF) est déclaré vainqueur dans tous les médias continentaux le surlendemain des élections, mais les résultats officiels scellent la victoire du président sortant, Salmin Amour, avec seulement 50,2 \% des voix. En 2000, les élections sont caractérisées par des fraudes patentes et la marge de victoire du CCM, représenté par Amani Abeid Karume, est de seulement 0,4 point. Enfin, en 2005, de nombreuses fraudes sont de nouveau rapportées. Les résultats officiels annoncent la réélection du président sortant, Karume, avec 53,2 \% des voix contre $46,1 \%$ à Hamad.

politiques ont abouti à la proposition de former un gouvernement dit « d'unité nationale ${ }^{7}$ devant amorcer le partage du pouvoir ${ }^{8}$. Ce gouvernement de 
coalition, entériné par un référendum, devait mettre $n$ à quinze années de toute-puissance de l'ancien parti unique en régime multipartite et de climat politique et social délétère. Le relâchement de la rivalité farouche entre les grandes figures politiques du pays s'est répercuté positivement sur la population. L'effervescence populaire qui a accompagné la campagne électorale n'a aucunement débouché sur les violents affrontements connus auparavant ni sur l'intervention armée des forces de l'ordre, mais s'est déroulée dans le calme et la bonne humeur. Si l'opposition est amère de voir son candidat échouer pour la quatrième fois et soupçonne de nouveau l'existence de fraudes électorales, le fair-play est de mise au lendemain d'élections qui ont vu le candidat du CCM, Ali Mohamed Shein, l'emporter de justesse avec 50,1 \% des voix contre 49,1 \% pour Seif Sharif Hamad ${ }^{9}$.

Malgré la nouveauté du climat politique de cette année électorale, les scores très serrés des candidats à la présidence de Zanzibar témoignent toutefois de la reconduction de divisions politiques anciennes, qui superposent aux affiliations partisanes des référents identitaires différents et des conceptions alternatives de l'appartenance à la nation. En effet, les enjeux sous-jacents aux tensions politiques incessantes, à savoir les relations entre les différentes populations des îles, entre Pemba et Unguja, et entre Zanzibar et l'Union, sont ancrés dans l'histoire des îles. Mais ces enjeux n'ont jamais été abordés de front parce qu'ils remettraient en question le grand récit national sur lequel s'est bâtie et a été reconduite la légitimité des élites au pouvoir ${ }^{10}$. Deux principales conceptions de l'identité nationale et de la légitimité souveraine prennent racine dans le paysage politique zanzibari avant 1964. D’un côté, le ZNP (Zanzibar Nationalist Party, « Parti nationaliste zanzibari ») prônait un nationalisme insulaire inscrit dans une culture swahilie musulmane et métissée, mais était réticent à reconnaître les droits politiques des descendants d'esclaves ou des travailleurs africains. De l'autre, l'ASP (Afro-Shirazi Party, « Parti afro-shirazi ») appelait les populations indigènes des îles, les Shirazis, et les nouveaux arrivants issus du continent à se

7. La terminologie peut prêter à confusion: bien qu' appelé gouvernement d'unité «nationale», il s'agit bien d'une coalition qui concerne le seul gouvernement de Zanzibar, et non celui de la République unie de Tanzanie ayant pourtant seule le statut de «nation » de jure.

8. Seuls les partis représentés à la Chambre des représentants participent au gouvernement d'unité nationale. Hormis le CCM et le CUF, aucun des autres partis en lice n'a obtenu suffisamment de voix pour pouvoir entrer au gouvernement.

9. Les candidats à la présidence des autres partis en compétition se partagent les $0,8 \%$ restants. 10. J. Oloka-Onyango et M. Nassali, Constitutionalism and Political Stability in Zanzibar. A Report of the Facts Finding Organised under the Auspices of the Kituo Cha Katiba, Dar es Salaam, KCK, 2003. 
libérer de ce qu'il décrivait comme le joug arabe du sultanat ${ }^{11}$. L'ASP prend le pouvoir après la Révolution du 12 janvier 1964, qui renverse le premier gouvernement indépendant du sultanat constitutionnel de Zanzibar formé le 10 décembre 1963. L'événement est interprété comme le renversement du pouvoir tyrannique d'une minorité arabe étrangère par une majorité africaine ${ }^{12}$. Depuis, le parti au pouvoir, l'ASP, rebaptisé CCM en 1977, renouvelle sa légitimité en réaffirmant sa délité à l'esprit de la Révolution, et assimile ouvertement le CUF à $\mathrm{l}^{\prime}$ ancien ZNP ${ }^{13}$. Les partisans du CCM ont intégré cette grille de lecture de la vie politique contemporaine et perçoivent les deux partis d'aujourd'hui comme la réincarnation des partis prérévolutionnaires. Par ailleurs, depuis 1964, l'île de Pemba a été marginalisée politiquement et économiquement par le centre de décision basé à Unguja parce que les populations de Pemba revendiquent plus fortement leurs origines arabes, que le Pemba prérévolutionnaire était largement acquis au ZNP et qu'il n'a pas pris part à la Révolution de 1964. Il n'est pas surprenant que depuis 1995, l'île vote en bloc pour le CUF, Seif Sharif Hamad étant par ailleurs originaire de Pemba, comme beaucoup de membres fondateurs de ce parti. Enfin, le dernier enjeu qui pèse de tout son poids dans la vie politique insulaire, à savoir la place de Zanzibar dans la République, est lié aux modalités de mise en œuvre du traité d'Union. Le 26 avril 1964, cent jours après la Révolution, la République du Tanganyika et la République populaire de Zanzibar signent un traité d'Union qui instaure la République unie de Tanzanie. Zanzibar conserve un gouvernement indépendant mais cède à l'Union les affaires régaliennes telles la défense, l'intérieur, les affaires étrangères ou les douanes. Du côté de Zanzibar, l'Union est présentée, à sa création, comme une structure qui devait permettre d'assurer le développement économique et social des îles. Mais, au 1 des ans, les populations insulaires aussi bien que la classe politique s'agacent de l'empiétement de la République tanzanienne sur les prérogatives insulaires et de leur marginalisation économique ${ }^{14}$. Ces tensions incessantes favorisent la recrudescence d'un sentiment national insulaire et l'activisme d'un mouvement autonomiste. Une plus grande indépendance de Zanzibar, que la mise en place d'un système fédéral à trois gouvernements pourrait rendre possible, est revendiquée par le CUF, tandis que le CCM reste prudent sur cette question d'une refonte de l'Union. Tout aussi désireux de conserver une large marge d'autonomie, le parti au pouvoir à Zanzibar sait en effet devoir sa force et son maintien au soutien du continent.

11. J. Glassman, «Sorting Out the Tribes. The Creation of Racial Identities in Colonial Zanzibar's Newspaper Wars», Journal of African History, vol. 41, 2000, p. 395-428. 12. M. F. Lofchie, Zanzibar: Background to Revolution, Princeton, Princeton University Press, 1965. 13. G. Cameron, « Zanzibar's Turbulent Transition », Review of African Political Economy, n 92, 2002, p. 313-330. 
14. H. Othman et C. M. Peter, Zanzibar and the Union Question, Zanzibar, Zanzibar Legal Services Centre, 2006.

\section{Des inscriptions électorales agitées}

Les inscriptions sur les listes électorales ${ }^{15}$, organisées sous la tutelle de la commission en charge du processus électoral dans les îles, la ZEC (Zanzibar Electoral Commission), débutent en juillet 2009 à Pemba et en septembre 2009 à Unguja ${ }^{16}$. Pour pouvoir s'inscrire, tout nouvel électeur est tenu de présenter un seul et unique document: sa carte d'identité zanzibarie (ZID) ${ }^{17}$. Pour les électeurs déjà inscrits, la carte d'électeur de 2005 constitue une pièce d'identité suf sante. Les formulaires de demande de cartes d'identité doivent être avalisés par le représentant local des autorités de Zanzibar, le sheha. Dans les premiers temps du processus d'enregistrement, les observateurs nationaux et internationaux constatent cependant que la plupart des shehas recourent à différentes manœuvres pour ne pas retourner les formulaires, prétextant le manque de temps, demandant un pot-de-vin, ou exigeant des pièces admi- nistratives non requises. En outre, des centaines d'anciens électeurs ne pouvant présenter leur ZID se voient éconduire. Parmi eux, nombreux sont ceux qui détiennent un bordereau de délivrance de ZID, mais qui ne sont jamais allés retirer leur carte. Lorsqu'ils se déplacent pour le faire, ils s'entendent dire que leur bordereau est devenu illisible et qu'ils doivent déposer une nouvelle demande, que leur carte n'est pas encore arrivée, ou encore que, retournée aux quartiers généraux de la ZEC, elle ne peut être renvoyée au bureau à temps ${ }^{18}$. Pointés du doigt par

15. Les observations rapportées dans ce paragraphe ont été menées par l'assistant de recherche. Elles ont été confrontées aux rapports des observateurs électoraux nationaux et internationaux. Voir notamment Tanzania Election Monitoring Committee, Temco Newsletter, $\mathrm{n}^{\circ} 1$, novembre 2009, et les rapports fouillés de l'International Law and Policy Institute (Ilpi), disponibles sur <tz.undp.org>. 16. À chaque nouvelle élection, le registre électoral, appelé Permanent Voters' Registrer (PVR), est mis à jour.

17. L'article 12(1) de la loi électorale de Zanzibar (Zanzibar Election Act) de 1984 révisée dispose $\mathrm{qu}^{\prime}$ « aucune personne ne doit être inscrite comme électeur si elle ne possède pas une carte d'identité délivrée comme énoncé dans la loi nº 7 de 2005 ». Pour pouvoir participer aux élections, il convient d'être de nationalité zanzibarie, d'avoir plus de 18 ans, et d'avoir résidé au moins trois ans dans une même circonscription électorale.

18. Voir Temco, Domestic Observation Mission : 2010 Zanzibar Election. October, 31 2010. Interim state-ment, Dar es Salaam, 2010, ainsi que les rapports de l'Ilpi, disponibles sur <tz.undp.org>. 19. Voir la déclaration conjointe des chefs de mission en Tanzanie de l'UE, de la Norvège, des États-Unis, du Canada et du Japon : Statement by Heads of Mission in Tanzania on the Voter Registration Process in Zanzibar, 13 août 2009, disponible sur <tanzania.usembassy.gov>. 
plusieurs représentations diplomatiques ${ }^{19}$, les freins posés à l'obtention des documents nécessaires pour s'inscrire sur les listes électorales, que des périodes de suspension des inscriptions ont tenté d'aplanir, semblent annoncer que les élections à venir vont se dérouler dans un climat tendu, si ce n'est violent.

Ces obstacles laissent aussi clairement apparaître l' «autorité discrétionnaire» 20 des shehas dans la conduite des processus électoraux. En tant que représentants des autorités insulaires, tous les shehas sont en effet des membres du parti au pouvoir. À chaque nouvelle élection depuis la réintroduction du multipartisme, la neutralité et l'impartialité des shehas aux différentes étapes du processus électoral à Zanzibar sont fortement remises en question. Dans les premières semaines d'inscription électorale à Pemba en 200921, le CUF s' est publiquement opposé au critère légal exigeant que soit présentée une carte d'identité pour pouvoir s'inscrire. Il arguait que de nombreux électeurs, de tout bord, n'avaient pas ce document en leur possession et ne pouvaient l'obtenir dans les délais impartis. Deux semaines seulement après le début du processus d'enregistrement des électeurs à Pemba, des manifestations sont organisées autour des bureaux des shehas pour réclamer la remise des formulaires de demande de ZID. Dans certains villages, des menaces sont proférées contre des shehas et des représailles violentes menées contre eux: acide jeté au visage ; passages à tabac nocturnes ; maisons et biens incendiés durant la nuit ; bêtes égorgées et champs brûlés. Si les shehas se voient refuser la protection rapprochée qu'ils réclament, le déploiement de forces de l'ordre dans de nombreux bureaux permet d'assurer leur sécurité physique tout en facilitant la poursuite de leurs manœuvres électorales.

Par ailleurs, les électeurs doivent s'armer de patience face à la lenteur du processus et à la confusion générale qui règne dans les bureaux. Informatisés, les enregistrements sont fortement ralentis et parfois reportés au jour suivant en raison d'incessants problèmes techniques, alors que la foule des électeurs, parfois venus de loin et partis très tôt de chez eux, attend avec impatience de pouvoir rentrer. En outre, ce ne sont pas toujours les mêmes documents qu'il convient de présenter selon les bureaux, de sorte que les électeurs repartent chez eux pour chercher un certi cat de naissance, une attestation de domicile ou un document faisant of ce de carte d'identité, tel un reçu d'inscription dans un hôpital ou une carte professionnelle. Il arrive que certains officiers acceptent $d$ 'inscrire des électeurs sur la seule bonne foi de témoins. L'exaspération face à ces obstacles,

20. Union européenne, Mission d'observation électorale, Preliminary Statement: General ElectionsTanzania 2010, 2 novembre 2010, p. 2. 21. L'obstruction à l'inscription des électeurs est plus fréquente à Pemba, acquise à l'opposition, qu'à Unguja. 
déjà à son comble en raison des problèmes de délivrance des cartes d'identité, déclenche de nouveaux actes de sabotage, attribués aux militants du CUF, qui sont destinés à effrayer la population et les officiers de la ZEC, et in ne à retarder voire à suspendre les inscriptions. À Pemba, des bureaux sont vandalisés; des nids de guêpes suspendus à la charpente; des œufs pourris cassés à même le sol; des matières irritantes éparpillées sur le matériel électoral. Autour de certains bureaux, la présence de forces armées, équipées de matraques, de fusils et de gaz lacrymogènes, effraie la population rassemblée. Dans les villages de l'île où les tensions sont à leur comble, des villageois décident d'organiser des rondes de nuit et certains individus, craignant des représailles, dorment en forêt. Les mauvais souvenirs des élections précédentes ont indéniablement été réveillés par ces pratiques d'intimidation. On dit officieusement, notamment parmi les militants du CUF, que près de 15000 personnes auraient été privées de leur droit de vote, sur un total de 407658 électeurs finalement inscrits pour prendre part aux élections.

\section{Du rapprochement des partis au gouvernement d'unité nationale}

Le rapprochement qui s'opère en plein milieu du processus d'inscription électorale entre le président sortant Amani Abeid Karume, et le chef de l'opposition, Seif Sharif Hamad 22, en novembre 2009, vient pourtant relancer l'espoir que l'année 2010 puisse finalement être celle de la pacification et de la démocratisation à Zanzibar ${ }^{23}$. Le 5 novembre, les deux leaders se rencontrent en secret au palais présidentiel. Le lendemain, les rumeurs vont bon train sur cette entrevue entre deux hommes qui se tiennent mutuellement en respect depuis des années. La stupeur laisse place à des espoirs de changements radicaux dans les îles lorsqu'on constate que la rencontre inaugure une détente (maridhiano en swahili) entre les partis. Pour la première fois depuis quinze ans, l'opposition reconnait la légitimité du gouvernement en place et de son président. À la mi-décembre, la nomination par Karume de deux représentants du CUF à la Chambre des représentants, Juma Duni Haji et Nassor Ahmed Mazrui, scelle le rapprochement voulu par le sommet du pouvoir. Les réactions sont mitigées à l'annonce du fait que ce rapprochement pourrait inaugurer la mise en place d'un

22. Ancien Premier ministre sous la présidence d'Aboud Jumbe entre 1985 et 1988, mais écarté du parti parce que favorable à la libéralisation de l'économie et à une autonomie accrue des îles, Hamad compte parmi les fondateurs du mouvement Kamahuru, devenu le CUF en 1992. 23. « What Next After Karume and Maalim Seif U-turn ? », Daily News, 29 novembre 2009.

gouvernement d'unité nationale, qui sera soumise à plébiscite. Les partisans du CUF sont prompts à saisir l'intérêt que leur parti gagnerait à participer au 
gouvernement: pour la première fois, l'opposition pourrait impulser des changements de l'intérieur. Mais, chez les radicaux du CCM, qui refusent depuis quinze ans tout dialogue avec l'opposition, la colère gronde. L'idée circule tout d'abord que les élections pourraient être repoussées d'un ou deux ans et Karume rester au pouvoir pour organiser, avec l'opposition, une transition démocratique. Certains n'y voient qu'une simple manœuvre autoritaire, d'autres une tactique visant à retarder l'échéance des procès pour corruption, détournement de fonds et usage privé de biens publics qui semblent attendre le président une fois son immunité levée. Lors de la $46^{\mathrm{e}}$ commémoration de la Révolution organisée à Pemba le 12 janvier 2010, Karume dément cependant les rumeurs de son maintien au pouvoir. Malgré le soutien apporté à Hamad, l'incompréhension règne chez les supporters du CUF qui voient leur leader assister pour la première fois aux célébrations d'une Révolution qu'ils refusent de reconnaître comme l'événement fondateur de la nation. Le 14 janvier, Seif Sharif Hamad prononce une allocution destinée à rassurer ses partisans, dans laquelle il argue de la nécessité de travailler ensemble dans une île politiquement déchirée depuis des décennies. Quant aux petits partis politiques, évacués de la scène, ils s'inquiètent d'une maridhiano strictement bipartite qui scelle leur marginalisation. Le 30 mars, la Chambre des représentants entérine le principe de la mise en place d'un gouvernement d'unité nationale.

L'idée d'instaurer le partage du pouvoir n'est pas nouvelle à Zanzibar. Au lendemain des premières élections multipartites de 1995, le président de l'Union sortant, Ali Hassan Mwinyi, et l'ancien président Julius Nyerere avaient insisté sur la nécessité d'une sortie de crise par la mise en place d'un gouver- nement de coalition $^{24}$. Mais aussi bien l'opposition que les membres du gouvernement $s^{\prime} y$ étaient déclarés fortement hostiles. La réconciliation était alors impossible car les divergences entre partis renvoyaient à des animosités farouches entre leaders ${ }^{25}$. Les échecs des différents accords de coopération (nommés muafaka en swahili) passés entre le CUF et le CCM en 1999, 2001 et 2008, sous la pression de l'Union, de la communauté internationale et de la société civile, qui visaient à prévenir les con its et les violences partisanes par la mise en place de réformes constitutionnelles destinées à assurer une plus grande indépendance de la justice et à favoriser la dépolitisation des médias, de la police et de l'administration

24. A. Crozon, «Zizanie à Zanzibar : les élections d'octobre 1995 », Politique africaine, $\mathrm{n}^{\circ} 61$, mars 1996, p. 133-138. 25. A. Babu, «Wanted: A Third Force in Zanzibar Politics», in H. Othman (dir.), Babu: I Saw the Future and It Works, Dar es Salaam, E \& D, 2001, p. 74.

civile, rendent compte des difficultés des deux partis à s'entendre sur les voies de la paci cation et de la démocra- tisation. Certes, ces accords aboutissent à la modi 
cation des procédures électorales, à l'établissement d'une commission électorale supposée indépen- dante et non partisane (la ZEC) et d'un registre électoral permanent (le PVR). Mais les tentatives de rapprochement s'enlisent après chaque nouvelle élection, le CUF refusant de traiter avec un parti prêt à tout pour conserver le pouvoir. Finalement, le pragmatisme prend le pas sur les inimitiés personnelles. Mais, contrairement à l'opinion selon laquelle le rapprochement est une initiative strictement zanzibarie, il semble que l'Union, principalement via son président Jakaya Kikwete, ait joué un rôle prépondérant dans le rapprochement entre les leaders zanzibaris. Ce dernier avait en effet publiquement promis, lors de sa première investiture en 2005, puis lors de la rencontre de Butiama entre le CUF et le CCM en 2008 visant à discuter les voies d'une sortie de con it, et en $\mathrm{n}$ lors d'une visite of cielle rendue au président américain Barack Obama en mai 2009, de régler les dif cultés politiques de Zanzibar, à savoir la violation des principes électoraux qui entachent la réputation de la Tanzanie dans son ensemble et limitent l'aide internationale accordée aux îles.

La surprise est d'abord grande, à la mi-juillet, de voir Ali Mohamed Shein, vice-président de l'Union, en fonction depuis 2001, choisi par le comité national exécutif du CCM pour porter la candidature du parti à Zanzibar. Mais sa tempérance et sa délité à Kikwete en font un candidat parfait pour le continent puisque la présidence de l'Union pourra continuer à peser de tout son poids sur les affaires des îles. Le parti semble aussi considérer que son origine - Shein vient de Pemba - peut permettre d'apaiser les différends entre Pemba et Unguja. Son handicap majeur est sa faible popularité : on lui reproche d'avoir mené sa carrière politique sur le continent et son absence de prise de position ferme sur la question du statut de Zanzibar et de la révision des termes de l'Union, ainsi que sur l'enjeu de la propriété des ressources pétro- lières offshore de Zanzibar. Son manque d'éloquence et son air débonnaire sont aussi pointés comme des défauts, notamment par rapport à la verve et au dynamisme des deux autres candidats du CCM en lice, Mohamed Gharib Bilal et Shamsi Vuai Nahodha, respectivement ancien Premier ministre et Premier ministre en fonction au moment des élections ${ }^{26}$. C'est surtout Bilal qui, fort du soutien de sa faction au sein du parti à Zanzibar, est plébiscité par les partisans du CCM. Pour qu'il ne disparaisse pas totalement de la scène en cette année 2010, il est choisi par le président de Tanzanie Jakaya Kikwete, candidat à sa réélection en Tanzanie continentale, pour être son colistier.

26. Shamsi Vuai Nahodha est désormais ministre de l'Intérieur au sein du gouvernement d'union. 


\section{Plébiscite populaire pour le partage du pouvoir}

L'enjeu du référendum organisé le 31 juillet 2010 pour décider de l'adoption du gouvernement d'unité nationale est crucial : il s'agit de savoir si les Zanzibaris sont prêts à ouvrir une nouvelle ère politique, économique et sociale. Le CUF, bien conscient qu'il a tout à gagner à participer au gouvernement, est leader de la campagne pour le « oui » qui débute vers la mi-juillet. Il tient de nombreux meetings pour appeler ses supporters à se rendre aux urnes et rappeler les procédures exactes de vote a n de limiter les bulletins nuls. Les partisans CCM du «non », qui tentent d'entrer dans le jeu en arguant que la loi interdit de mener campagne avant la tenue d'un référendum, se font rabrouer par la faction CCM qui soutient le rapprochement initié par Karume. À seulement quelquesjoursduréférendum,cecamps'engagefortementdanslacampagne: villes, bourgs et villages sont placardés d'af ches et des tracts inondent les places publiques. Le 30 juillet, Amani Karume lui-même, dans une allocution télévisée, appelle la population à voter « oui » en bloc pour que règnent en $n$ la paix et la tranquillité sur les îles. Les radicaux du CUF du maskani ${ }^{27}$ de Jaws Corner, une place ouverte au cœur de Stone Town, semblent tendre la main au CCM : sur leur tableau noir était placardée, les 30 et 31 août, l'af che jaune et verte du CCM portant les photos de sept personnalités du CCM à Zanzibar et appelant à voter «oui». Pendant toute cette période, la campagne pour le «non » doit se faire discrète, tant la pression des partisans du « oui » est forte. Elle passe par des messages téléphoniques, du porte-à-porte ou de petits meetings de quartiers. Cette campagne souterraine, menée par les partisans déçus du candidat Mohamed Gharib Bilal, est soutenue par différents argu- ments : la participation du CUF au gouvernement limitera le pouvoir du CCM ; les mésententes qui ne manqueront pas de surgir entre les partis viendront attiser les tensions plutôt que les atténuer ; la présence de deux leaders origi- naires de Pemba entraînera une augmentation des fonctionnaires originaires de Pemba dans les services publics, et en contrepartie la marginalisation voire la discrimination de ceux originaires d'Unguja. Pendant deux semaines, au maskani de Kisonge, dans le quartier de Michenzani, ef de Mohamed Gharib Bilal, on peut lire, écrit à la craie sur le tableau noir planté au bord de la route : «Partisans du CCM, le gouvernement de coalition est dangereux: au réfé- rendum votez non, non, non! ». Amani Karume subit directement des attaques personnelles: à quelques jours du référendum, une photo originale du pré- sident encore enfant posant avec des membres de sa famille, qui ornait ce

27. Les maskani désignent les lieux, baraques construites en dur ou simples bancs en bois portant les couleurs et le drapeau du parti, où se retrouvent les sympathisants d'un parti pour discuter de politique. Ils ont été créés dans les années 1990 après l'adoption du multipartisme. 
même maskani depuis plusieurs années et à laquelle le président tenait particulièrement, est dérobée. Craignant pour sa sécurité personnelle et celle de sa famille, il renforce la surveillance du palais présidentiel ${ }^{28}$.

Tous les partis politiques, cependant, ne se sont pas joints à la campagne du référendum, que ce soit pour le «oui» ou le «non». Ainsi, pour Jahazi Asilia (le «boutre authentique», formé en 2004), qui défend la souveraineté des îles par l'instauration d'un gouvernement indépendant à Zanzibar lié au Tanganyika par un nouveau traité ( $d^{\prime}$ association et non d'union), les résultats du référendum importent peu dans la mesure où ils ne s'accompagneraient aucunement d'une modi cation en profondeur de la constitution. Selon le président de ce parti, Amour Rajab Amour, ancien fonctionnaire et ancien membre fondateur du CUF, le gouvernement des îles est une autorité fantoche dirigée en sous-main par le gouvernement de l'Union. Par ailleurs, arguant de sa longue expérience du fonctionnement interne du CUF et de la fré- quentation de ses membres les plus en vue, il souligne que la détention de positions de pouvoir par le CUF n'entraînera aucun changement fondamental, les membres de ce parti aspirant bien plus, selon lui, aux avantages nanciers et statutaires que leur offre le pouvoir qu'au bien commun. En n, puisque les petits partis d'opposition ne parviennent jamais à obtenir de siège à la Chambre des représentants, ils ne seront pas représentés dans le nouveau gouvernement et ne pourront avoir aucune in uence sur le destin des îles. Le même type d'argumentaire est convoqué par les autres partis d'opposition pour soutenir que le nouveau système de gouvernement ne serait qu'un miroir aux alouettes.

Le $1^{\text {er }}$ août, les résultats du référendum sont annoncés par la ZEC à la radio et à la télévision : 66,4 \% de la population ont voté « oui ». Les supporters du CUF sont ravis. Au «salon urbain ${ }^{29}$ » de Jaws Corner, le tableau noir a été débarrassé de l'af che de campagne du CCM et, à sa place, est inscrit à la craie, en gros chiffres, le résultat du référendum. De leur côté, les partisans du«non»décomptentlesbulletinsàleurmanièrepoursoutenirqu'enréalité, c'est leur camp qui l'a emporté : additionnant les voix du «non », les bulletins nuls qui exprimeraient en fait le désaccord des votants, et les abstentionnistes qui sont eux aussi considérés comme des partisans du non, ils déclarent que le «non» l'emporterait à près de $52 \%{ }^{30}$. Le 10 août, la Chambre des repré- sentants entérine le changement de système politique avant d'être dissoute

28. «Tanzanie : Amani Karume craint pour sa sécurité », La lettre de l'océan Indien, n 1291, 28 août 2010. 29. M. Chauvin, "Jaws' Corner, salon urbain, palpitations insulaires », in N. Bernardie-Tahir (dir.), L'Autre Zanzibar. Géographie d'une contre-insularité, Paris, Ades-Dymset-Geolab-Karthala, 2008, p. 63-67. 
30. Temco, Temco Newsletter : Special Issue on Zanzibar Referendum, $\mathrm{n}^{\circ}$ 5, juillet 2010.

dans l'attente des prochaines élections. Non seulement le principe d'un gouvernement d'unité nationale est-il adopté dans le $10^{\mathrm{e}}$ amendement à la Constitution, mais il est aussi désormais inscrit dans la Constitution que Zanzibar est un nchi, terme signi ant «pays» mais que les Zanzibaris assi- milent aussi à «patrie» et à «nation». Les réactions du continent ne se font pas attendre. Plusieurs organismes et des avocats indépendants prennent la parole pour af rmer qu'un tel changement constitutionnel doit être entériné par l'Union. Ils craignent que cette revendication de souveraineté mène, à long terme, au démantèlement de la République.

\section{Les tensions contenues de la campagne électorale}

La campagne électorale débute à la mi-septembre, peu après la $\mathrm{n}$ du ramadan. Les candidats à la présidence sillonnent les îles pour se présenter aux électeurs et exposer le programme de leurs partis. Les enjeux cruciaux que constituent l'Union et l'exploitation des ressources pétrolières des îles sont évacués des meetings du CCM et ne sont abordés qu'avec prudence par le CUF, alors que certains petits partis, tel Jahazi Asilia, en font leurs thèmes de campagne. Plutôt que d'exposer une vision politique générale à l'échelle de l'archipel, les candidats du CCM et du CUF se contentent de promettre le développement et l'amélioration des infrastructures des îles, région par région et village par village. Les discours se déclinent autour des thèmes de la construction de routes, d'écoles et d'hôpitaux, et de l'approvisionnement en eau et en électricité. Ali Mohamed Shein fonde sa campagne sur l'énumération des actions concrètes de développement qui seront menées localement. Ainsi, lors de deux meetings tenus dans des bourgs voisins de la côte Est et Sud-Est, l'un à Paje le 10 octobre, l'autre à Makunduchi le 22 octobre, le candidat du CCM à la présidence de Zanzibar rappelle à son auditoire les actions déjà accomplies dans la région, citant les portions de routes construites sous la présidence de Karume et les villages reliés à l'eau ou à l'électricité. Il poursuit en indiquant le nombre d'écoles et de résidences professionnelles pour enseignants qui seront construites au cours $\mathrm{du}$ quinquennat à venir en citant les villages précis où elles seront implantées, et en mentionnant les nanceurs : la Banque mondiale et les fonds d'aide au développement des Émirats arabes unis. Le CCM s'approprie ainsi des projets à venir déjà négociés par le gou- vernement en place pour en faire des promesses de campagne, démontrant par là même la quasi-fusion entre ces deux structures.

Le CUF, n'ayant jamais détenu le pouvoir, ne peut ni s'enorgueillir de ses actions passées ni énumérer les mesures concrètes à venir, lieux, délais et nanceurs à 
l'appui, comme le fait son opposant. Il fonde alors symboliquement son pouvoir sur la remise, en début de meeting, des cartes de parti à ses nou- veaux adhérents appelés un à un à venir les retirer des mains de Seif Hamad. Plus porté à saisir l'occasion des meetings de campagne pour développer une vision de la nation zanzibarie ère et autonome, le parti ne recule cependant pas devant les promesses aisément comprises. Les propos tenus par Hamad lors d'un meeting organisé le 20 octobre à Kizimbani, dans la circonscription de Dole, une zone fertile située au centre d'Unguja et parsemée de grandes plantations, illustrent la mobilisation du thème du ventre bien rempli :

«Combien parmi vous boivent du lait, hein ? [...] Aujourd'hui le lait est devenu un aliment de luxe réservé à quelques personnes [...]. Nous voulons construire un nouveau Zanzibar, construire un nouveau Zanzibar où celui qui veut manger de la viande mange de la viande, d'accord? Si tu veux du poulet, tu manges du poulet. Si tu veux de la viande de chèvre, tu manges de la viande de quoi ? De chèvre!».

Le nouveau Zanzibar est réduit à un gouvernement gestionnaire de la faim. La facilité de ces propos n'échappe pas aux partisans du CCM, qui reprennent ironiquement l'image du lait dans certains de leurs slogans. Mais ce discours témoigne aussi de la stratégie politique du CUF: attirer à lui les déçus du CCM et les populations économiquement marginalisées, et se débarrasser de l'image de parti représentant uniquement une section de la population, à savoir les gens de Pemba et les groupes d'origine étrangère.

Il est intéressant de constater que les référents culturels zanzibaris revisités pour l'occasion sont fortement mobilisés pendant la campagne, sous leur forme matérielle ou immatérielle. La tribune du CUF est une structure en bois sculpté et incrusté de clous dorés qui imite les coffres traditionnels. Le CCM débute chacune de ses réunions par des utenzi, longs poèmes chantés qui font partie de la tradition épique swahilie, ici déclamés par des jeunes lles louant le parti et le candidat en lice. Et aussi bien les réunions du CUF que celles du CCM sont ouvertes par la bénédiction d'un imam. Mais certains référents ne sont pas partagés par les deux partis. Lors d'un meeting du CUF tenu le 28 octobre dans la vieille ville de Stone Town, sur la place Culture, les festivités qui ouvrent le rassemblement intègrent des danses hindoues effectuées par des jeunes lles de toutes origines vêtues de saris. Le mélange des cultures et la présence de la communauté indienne sont donc reconnus par le CUF comme des traits originaux de la culture zanzibarie. La référence historique à la Révolution apparaît, quant à elle, uniquement dans les meetings du CCM sous forme de slogan (l'orateur lançant d'une voix forte «Mapinduzi! » - «Révolution!» -, la foule lui répondant en chœur «Daima!» - «Toujours!») 
ou sur les bannières qui délimitent l'emplacement des invités d'honneur et du podium. Le 10 octobre à Paje, on pouvait ainsi lire : « Ne perds pas ton vote pour ceux qui combattent la Révolution ». Le nom des groupes de danse et de tambours qui divertissent la foule CCM contient souvent le terme d'Afro- Shirazi, du nom de l'ancien parti unique, l'Afro-Shirazi Party, ainsi baptisé en référence aux populations qu'il représentait sur les îles, à savoir les Africains et les populations indigènes nommées Shirazis. On voit donc que l'appropriation des éléments du patrimoine historique et culturel des îles est sélective, exprimant des conceptions radicalement différentes de l'identité nationale, l'une ancrée dans une culture swahilie métissée, l'autre tournée vers la culture négro-africaine continentale et s'af chant comme l'héritière de l'esprit de la Révolution. En n, chaque parti lance ses chansons de campagne créées par de jeunes artistes de la scène musicale locale. Le CUF sort vainqueur dans cette compétition musicale en raison du succès de ses deux tubes, entendus à chaque coin de rue en $\mathrm{n}$ de campagne, Hodihodi et Maalim Seifu Sharifu, le premier chantant une victoire déjà acquise («Toc Toc/Nous entrons au palais présidentiel»), le second vantant la popularité du leader du CUF et sa reconnaissance internationale (« Notre choix c'est toi/Maalim Seif Sharif/ Tous les votes pour toi/Que le monde entier nous complimente »).

À l'approche des élections, les meetings de campagne rassemblent une foule toujours plus importante. Du côté du CCM, la distribution gratuite de tee-shirts, de pagnes, de casquettes, parfois de tenues de sport complètes aux couleurs du parti, et la gratuité des transports organisés pour se rendre aux meetings ne sont pas étrangères à cette forte mobilisation. Les réunions prennent une véritable tournure festive: les allocutions des personnalités politiques s'effacent derrière les performances musicales, les jeunes lles troquent leur voile ou leur hidjab contre tee-shirt et jean moulant, et la jeunesse s'amuse en groupes mixtes loin de la tribune et des haut-parleurs. Les meetings du CUF sont plus encadrés et organisés. Non seulement les partisans doivent s'offrir eux-mêmes des vêtements aux couleurs du parti et couvrir leurs frais de déplacement, mais la mixité est contrôlée : dans les meetings, il est maintes fois répété que les femmes doivent s'asseoir à l'avant, non loin du podium, vêtues des pagnes du CUF, tandis que les hommes restent debout à l'arrière ; quant aux transports, ils sont, autant que possible, organisés par sexe. L'effervescence qui gagne une partie de la population éclipse, à deux semaines du scrutin, la mise à disposition publique des listes électorales. Cette mesure doit permettre à la ZEC et aux représentants des partis de véri er que des électeurs ne sont pas inscrits dans plusieurs localités. Les fraudeurs identi és sont privés de leur droit de vote. En dépit de l'euphorie d'une partie de la population et des efforts de transparence des autorités 
publiques, les souvenirs

des élections précédentes sont encore présents : des familles font des réserves de nourriture, et certains déclarent qu'ils resteront enfermés chez eux jus- qu'aux résultats.

À deux jours du scrutin, la rivalité entre les partis s'exprime à travers l'appropriation de l'espace public au moyen de décorations. Au rond-point de Michenzani, où le fameux maskani CCM de Kisonge, bigarré aux couleurs du parti, fait face au maskani CUF de Commonwealth, le CUF est le premier à installer de grandes guirlandes blanches et bleues. Le soir même, les partisans du CCM arrachent ces guirlandes pour installer les leurs. Les forces de l'ordre doivent intervenir. Le lendemain, les couleurs des deux partis se mêlent dans cet espace devenu, en cette année 2010, symbole d'une entente cordiale non dépourvue de tensions partisanes.

\section{Un scrutin sous influence}

Le jour du scrutin, les opérations électorales se déroulent dans l'ordre et dans le calme, avec un taux important de votants $(89,5 \%)$. Les bureaux de vote en ville et à la campagne n'ont jamais été aussi bien tenus ${ }^{31}$ : les policiers qui encadrent les écoles transformées ce jour-là en bureaux ne sont pas armés et paraissentdécontractés;lesélecteursattendentcalmementen le,àl'extérieur, d'être invités à entrer pour voter ; des représentants des partis politiques sont présents et véri ent l'identité des électeurs; les handicapés et les personnes âgées sont autorisés à se faire accompagner ; les électeurs, une fois leur tour passé, imprègnent leur index et leur auriculaire d'encre indélébile, ce qui leur interdit tout nouvel accès au bureau. Des observateurs nationaux de toute origine (partis politiques, médias, associations et $\mathrm{ONG}$ ) et des observateurs internationaux circulent de bureau en bureau. Les soupçons de fraude qui circulent pendant le vote - un fonctionnaire aurait pré-rempli des bulletins de vote, et des électeurs auraient réussi à se débarrasser de l'encre indélébile pour voter une seconde fois - attirent sur les lieux supposés de la fraude les journalistes et les observateurs. Des cas mieux documentés sont rapportés par des observateurs du CUF: des représentants de la ZEC auraient donné plus de bulletins de vote que nécessaire et les électeurs corrompus auraient

31. Voir les rapports des observateurs nationaux : Jumaza Observer Group, Ripoti ya awali ya uchaguzi mkuu wa Zanzibar Oktoba 31, 2010, Zanzibar, 2010; Temco, Domestic Observation..., op. cit. Voir également ceux des observateurs internationaux, notamment Union européenne, Mission d'observation électorale, Preliminary Statement..., op. cit. ; Southern African Development Community, Electoral Observers Missions to Tanzania, Preliminary Report of the 2010 Elections, Dar 
es Salaam, 2 novembre 2010.

glissé plusieurs bulletins dans les urnes; une femme aurait distribué des cartes d'électeurs; un militaire aurait fait pression dans un bureau de vote pour que vingt électeurs sans carte ni autorisation spéciale de la ZEC puissent voter. En n, ces mêmes observateurs af rment que les résultats af chés dans certains bureaux de vote ne correspondent pas aux résultats parvenus à la ZEC. Si le scrutin se déroule globalement dans le respect des droits des électeurs, c'est-à-dire sans pression apparente ni présence menaçante des forces armées, des doutes planent néanmoins sur le respect des règles électorales.

C'est surtout la lenteur de la ZEC à annoncer les résultats des élections qui contribue à soulever les craintes de manipulation électorale. Le soir du scrutin, à Jaws Corner, tout incident est interprété comme une tentative de tricherie. Les accusations de sabotage vont bon train lorsque la Zanzibar Cable Television, une chaîne de télévision qui diffusait en direct le décompte des bulletins de vote, est soudainement coupée. Des scooters passent régulièrement apporter les résultats partiels par circonscription, consciencieusement notés et âprement commentés pour savoir si le nombre de votes obtenus par Hamad lui permettra de remporter le scrutin. Vers 1 heure du matin, lorsque la rumeur qu'une manipulation de grande ampleur est organisée par le CCM se répand, le mot d'ordre est passé d'aller réveiller chaque maisonnée et d'organiser une manifestation dans les rues de la vieille ville. Mais le mouvement retombe bien vite: seule une dizaine de personnes vient grossir le petit groupe de sympathisants rassemblés pour la nuit. La grande majorité de la population, consciente que les résultats tarderont à être annoncés et échaudée par les violences passées, patiente à la maison.

Le lendemain des élections, au petit matin, la vieille ville est désertée. La population, prudente, reste sur ses gardes plusieurs heures avant de reprendre le cours habituel de ses activités. À l'hôtel de Bwawani où, depuis la veille au soir, la ZEC annonce aux journalistes et aux observateurs les résultats par circonscription au 1 du décompte des bulletins, une foule de partisans du CUF est rassemblée devant les grilles d'entrée. Ils passent la journée à scander en chœur les slogans de campagne, faisant pression pour que les résultats dé nitifs soient annoncés dans les plus brefs délais. Malgré l'autodiscipline, c'est aussi la forte présence des forces de l'ordre, armées et équipées de pied en cape, qui tient la foule en respect. Au 1 de la journée, la foule devient plus pressante mais paraît consternée par l'absence de toute annonce. À son arrivée à Bwawani en $\mathrm{n}$ de soirée, Seif Sharif Hamad rappelle au haut- parleur la consigne martelée pendant la campagne : il faut rester discipliné et accepter la voix des urnes. Parmi les journalistes et les observateurs rassemblés dans la salle climatisée de Bwawani, le 
scepticisme est de mise à l'écoute des

déclarations du président de la commission électorale, Khatib K. Mwinyichande, qui af rme que la lenteur de la proclamation des résultats est chose normale. Il se dit à voix basse qu'il ne fait plus aucun doute que les résultats sont truqués, et les observateurs internationaux s'agacent de se voir refuser l'accès des centres de validation de la $\mathrm{ZEC}^{32}$. La nouvelle circule aussi qu'une commission spéciale, comptant le président de Tanzanie, Jakaya Mrisho Kikwete, et les chefs de l'armée, serait venue du continent pour faire pression sur les membres de la ZEC. Lorsqu'à 21 h 30, le président de celle-ci annonce la victoire de Ali Mohamed Shein à 50,1 \% contre Seif Sharif Hamad à 49,1 \% des voix, les derniers espoirs de l'opposition sont anéantis. Encore une fois, l'écart entre les deux opposants est ténu, puisque seules 3471 voix les départagent. Dans un pays où l'on soupçonne que des centaines d'électeurs, pour la plupart des partisans du CUF, ont été refoulées lors de l'inscription électorale, les doutes planent sur la crédibilité de ces élections.

Après l'annonce des résultats, la foule rassemblée aux portes de Bwawani suit les appels au calme de Seif Hamad et se disperse sans remous tandis que les partisans du CCM se rassemblent à Kisonge pour fêter la victoire. Le lendemain, le lieu est pris d'assaut par une foule mêlant partisans du CUF et du CCM. Tous dé lent en trottinant par petits groupes et en scandant les slogans de campagne, arborant parfois les couleurs d'un seul parti, parfois celles des deux partis. Ces manifestations font écho aux propos tenus par Seif Sharif Hamad durant la campagne et le soir de l'annonce des résultats, à savoir qu'il n'y a ni vainqueur ni vaincu, mais que ces élections sonnent la victoire de tous les Zanzibaris. Si on est loin du vent de panique qui avait souf é sur les élections de 1995, avec le retour précipité des gens de Pemba dans leur île natale, tout comme de l'exil de l'opposition vers le Kenya en 2001, de nombreux supporters du CUF expriment pourtant leur déception. Observant avec scepticisme les manifestations d'euphorie de la jeunesse rassemblée à Kisonge, ils af rment rester convaincus que le scrutin a de nouveau été truqué.

L es résultats des élections de 2010 à Zanzibar illustrent derechef à quel point les divisions politiques se superposent à des clivages identitaires historiquement construits et reconduits jusqu'à aujourd'hui. Le CUF a encore une fois remporté l'ensemble des 18 circonscriptions de Pemba, et quatre des 32 circonscriptions à 
Unguja, l'une déjà tenue par le parti (Stone Town), les autres ayant longtemps recueilli un grand nombre de voix pour l'opposition (Magogoni, Mtoni et Nungwi). En raison de l'entente instaurée entre le CCM

143

32. Union européenne, Mission d'observation électorale, Preliminary Statement..., op. cit., p. 7.

et le CUF, et des appels du leader de l'opposition à faire bonne gure face aux résultats quels que soient les soupçons de fraude, l'atmosphère préélectorale et postélectorale est restée sereine. Non seulement les supporters de l'opposition se réjouissent de voir leur leader investi à la vice-présidence des îles ${ }^{33}$, mais ils s'approprient aussi la victoire. Alors que dans les jours qui suivent les élections, les supporters du CCM retombent dans le silence, de petites fêtes de quartiers sont organisées à Stone Town par des partisans du CUF pour célébrer l'entrée de leur parti au sein du gouvernement. Le jour de la prestation de serment du nouveau président Ali Mohamed Shein, le 4 novembre au stade Amaan, l'une des deux tribunes réservées à la popu- lation porte unanimement les couleurs du CUF. Au nom d'Hamad, prononcé par le nouveau président dans son discours de remerciements, elle retentit de cris de joie. Pourtant, les soupçons de fraude et les doutes sur le rôle joué par la République dans la reconduction du CCM au pouvoir laissent un goût amer à maints supporters du CUF. Ils suggèrent que la question posée par Ben Rawlence au sujet des élections de 2005 à Zanzibar, à savoir «jusqu' à quand le gouvernement de l'Union va-t-il aider l'administration de Zanzibar à conserver le pouvoir ? ${ }^{34} »$, est toujours d'actualité, alors même qu'au sein de la population, partisans CCM compris, le poids de l'Union dans la politique des îles est de plus en plus perçu comme une intrusion mal venue. Gage de démocratisation auprès de la communauté internationale, le principe du partage du pouvoir qu'entérine le gouvernement d'unité nationale a ceci de stratégique qu'il permet au CCM de se maintenir au pouvoir et d'affaiblir la charge critique de l'opposition, mais ne manquera pas d'entraîner un fort mécontentement populaire si les promesses de changement ne sont pas tenues (5)

Marie-Aude Fouéré Les Afriques dans le Monde (LAM) Sciences Po Bordeaux - Université Montesquieu-Bordeaux iv

33. Deux vice-présidents ont été désignés: le premier est Seif Sharif Hamad (CUF), le second Seif Ali Idd (CCM). 34. B. Rawlence, « Briefing... », art. cit., p. 522. 
